\title{
Germanica
}

\section{Les revues d'art viennoises de la fin de siècle ou comment construire une nation}

Étude de cas : la Hohe Warte de Joseph August Lux

Wiener Kunstreitschriften um 1900: die Nationen im kunstkritischen Diskurs.

Eine Fallstudie: Josef August Lux Hohe Warte

Sylvie Arlaud

\section{OpenEdition}

Journals

Édition électronique

URL : http://journals.openedition.org/germanica/588

DOI : 10.4000/germanica.588

ISSN : 2107-0784

Éditeur

Université de Lille

Édition imprimée

Date de publication : 1 décembre 2008

Pagination : 183-192

ISBN : 978-2-913857-22-6

ISSN : 0984-2632

Référence électronique

Sylvie Arlaud, "Les revues d'art viennoises de la fin de siècle ou comment construire une nation », Germanica [En ligne], 43 | 2008, mis en ligne le 01 décembre 2010, consulté le 06 octobre 2020. URL http://journals.openedition.org/germanica/588 ; DOI : https://doi.org/10.4000/germanica.588

Ce document a été généré automatiquement le 6 octobre 2020.

(c) Tous droits réservés 


\title{
Les revues d'art viennoises de la fin de siècle ou comment construire une nation
}

\author{
Étude de cas : la Hohe Warte de Joseph August Lux \\ Wiener Kunstreitschriften um 1900: die Nationen im kunstkritischen Diskurs. \\ Eine Fallstudie: Josef August Lux Hohe Warte
}

Sylvie Arlaud

1 Les revues d'art et de décoration sont autant les produits que les révélateurs des mutations profondes qui vont traverser les arts et les modes de vie européens au tournant du $\mathrm{Xx}^{\mathrm{e}}$ siècle. Tout comme dans les autres capitales européennes, à Vienne, les journaux et les revues spécialisées dans les arts et la littérature, les musées et les théâtres, connaissent un essor fulgurant sous l'influence libérale dès les années 1860 . Ils se développent comme des centres d'opinion en rivalité ou en collaboration avec le pouvoir dynastique ${ }^{1}$. Ces médias sont soumis à plusieurs contraintes, d'ordre politique bien sûr, mais avant tout d'ordre économique. Et c'est bien ce primat économique qui peut être vu comme la raison essentielle de cette alliance étonnante entre la modernité, l'avant-garde culturelle et l'État.

2 Contrairement aux revues élitistes, les revues d'arts décoratifs sont le lieu où se rencontrent vulgarisation esthétique et intérêt économique ${ }^{2}$. Dans le paysage des revues on retrouve la collusion d'intérêt entre le pouvoir établi et le contre-pouvoir libéral des nouveaux organes de presse. Notons qu'à Vienne l'organe officiel de la couronne autrichienne du Musée d'art et d'industrie, Kunst und Kunsthandwerk, est, à ses débuts en 1897, sous l'impulsion de son directeur Arthur von Scala, le chantre de la modernité et présente délibérément les produits Arts and Crafts de l'école Morris afin d'inspirer les élèves et les artisans locaux, affaiblis par la dissolution des corporations en 1859 et la crise économique de $1873^{3}$. Les articles et le programme ne se différencient pas fondamentalement de ce que l'on pourra lire au même moment dans l'organe de la Sécession viennoise, Ver sacrum ${ }^{4}$. De même, en Allemagne, la question de 
l'art et de l'artisanat devient une affaire d'État. Bode, Muthesius ou Lichtwark sont investis par le gouvernement pour assurer le rayonnement de la nation à travers les arts par la mise en place d'un programme d'expositions.

Avec les arts décoratifs, la scission entre pouvoir conservateur et contre-pouvoir moderniste se soumet à l'impératif économique ${ }^{5}$. Dans son ouvrage sur La création des identités nationales, Anne-Marie Thiesse explique que les nations tentent à tout prix de revaloriser l'artisanat, de le poser comme réponse adéquate à la révolution industrielle, où l'identité esthétique du produit reste garantie ${ }^{6}$. Ainsi, la création des musées d'art et d'industrie est liée de très près à deux formes émergentes de la modernisation: l'industrialisation et la naissance des nationalismes'. L'Angleterre ouvre la voie avec le South Kensington Museum en 1851 et sera imitée dix ans plus tard par deux pays, frères rivaux. Tout comme l'Angleterre a su dépasser son maître français ${ }^{8}$, l'Allemagne doit organiser ses écoles, ses musées pour gagner la bataille des arts décoratifs ${ }^{9}$, c'est ce que l'on peut lire sous la plume de Wilhelm Bode. Mais avant l'Allemagne, c'est l'Autriche qui emboite le pas à l'Angleterre dès 1863 avec la création du Musée d'art et d'industrie. Le Reich inaugure le Kunst und Gewerbemuseum à Berlin quatre années plus tard ${ }^{10}$. La quête identitaire des deux nations, dont l'émergence de l'une scelle la crise de l'autre, se lit ainsi à travers l'étude des arts décoratifs. Tout s'y cristallise: les mutations structurelles imprimées au paysage urbain et à la vie quotidienne, les changements sociaux et politiques, les ruptures dans la conception des arts, dorénavant ancrés dans le processus économique, profondément hétéronomes, alors même que certains mouvements esthétiques de la fin de siècle tentent de se retrancher dans une sphère autonome. Les musées et les revues d'arts décoratifs se développent comme les lieux où l'on tente d'opérer la très délicate création d'une identité nationale visible, exportable, dont le succès économique est le succédané des victoires militaires.

Dans le paysage déjà très saturé des publications d'art apparaît en 1904 une revue symptomatique des contraintes imposées aux artistes et aux critiques en Autriche. Son éditeur et principal contributeur, Joseph August Lux, permet de retracer les principales contradictions de cette modernité viennoise dans son rapport aux autres capitales européennes.

\section{La revue Hohe Warte dans l'édition d'art européenne}

Joseph August Lux (1871-1947), l'éditeur de la revue Hohe Warte, Illustrierte Halbmonatsschrift für die künstlerischen, geistigen und wirtschaftlichen Interessen der städtischen Kultur, publiée de 1904 à 1908, ne fait pas partie des grands noms de la critique esthétique viennoise. Et pourtant, deux études récentes ${ }^{11}$ ont fait ressortir sa grande force de travail. Il signe des articles pour de nombreuses revues à partir de 1900, dirige Die Fläche, puis la revue liée à la Wiener Werkstätte, Das Interieur, à partir de 1904. Il se décrit volontiers lui-même comme le médiateur principal des idées Arts and Crafts auprès des cercles créatifs viennois, et même comme le fer de lance de la Sécession. Mais ce jugement lui appartient entièrement. Un long voyage en Angleterre le familiarise avec les écrits de Morris et de Ruskin et lui permet d'apprécier l'efficacité didactique des expositions du South Kensington Museum. Cependant, lorsque paraît en novembre 1904 le premier numéro de la Hohe Warte, Hofmannsthal ${ }^{12}$ a déjà cité Ruskin depuis presque dix ans, Arthur von Scala s'est attiré les foudres des critiques dès 1898, Hermann Bahr et Adolf Loos ont déjà milité pour les Arts and Crafts dans la Neue Freie 
Presse ou Die Zeit et la Wiener Werkstätte vient d'être fondée en 1903 en s'inspirant explicitement du programme anglais de Ruskin et Morris.

6 Lux n'a donc pas fonction de précurseur, mais bien de vulgarisateur. De même est-il tout à fait conscient de lancer sa revue sur un marché saturé. Les revues d'art, si elles émergent à la fin du XVIII ${ }^{\mathrm{e}}$ siècle, vont véritablement se développer à la fin du XIX $\mathrm{e}^{\mathrm{e}}$ siècle : en 1884 paraît The Hobby Horse de Mackmurdo, trois années plus tard Der Kunstwart de Ferdinand Avenarius à Dresde. The Studio. An Illustrated Magazine of Fine and Applied Art $^{13}$, le modèle des revues d'art en Europe, voit le jour en 1893, Pan $^{14}$ de Julius Meier Graefe en $1894^{15}$. En 1897, Kunst und Kunsthandwerk est lancée à Vienne, Deutsche Kunst und Dekoration à Darmstadt et Dekorative Kunst à Munich. Enfin, Ver sacrum publie son premier numéro en 1898.

7 Quel est alors le rôle de cette nouvelle revue ? Contrairement à Kunst und Kunsthandwerk elle n'est pas liée à une institution, contrairement à Ver sacrum elle ne défend en apparence aucun mouvement esthétique précis, même si Lux est lié au cercle de la Wiener Werkstätte, dont le programme paraîtra d'ailleurs dans les pages de sa revue ${ }^{16}$. Le titre, Hohe Warte, fait référence à la colonie d'artistes et de mécènes située sur l'une des hauteurs de Vienne ${ }^{17}$, construite sur les modèles d'Olbrich et de Hoffmann. Mais la Hohe Warte se veut d'abord l'incarnation d'un idéal urbain, social et architectural, mais aussi un poste d'observation, dégagé de tout parti pris, d'où l'on peut jauger les créations étrangères et locales. Quel est alors le positionnement de sa revue sur l'échiquier des créations autrichiennes? Dans son avant-propos, l'éditeur et principal contributeur part d'un constat négatif ${ }^{18}$. S'il crée sa revue, c'est pour tenter de trouver une réponse à la décadence esthétique des grandes villes et à la perte des valeurs et des traditions qu'il croit observer en Autriche mais aussi en Allemagne ${ }^{19}$. Pour autant, il ne prône pas la supériorité de l'ancien sur le moderne, mais veut se tourner vers des études de cas précis, le plus souvent loin de la capitale, dans lesquels il oppose le bon au mauvais exemple. La démarche est didactique et clairement empruntée aux expositions du South Kensington Museum. Il s'adresse de fait au plus grand nombre à des fins d'éducation esthétique, sans distinction de classe ${ }^{20}$. Revenons à l'avant-propos:

In Geschmacksdingen bestehen heute nur sehr verworrene Anschauungen: vom Vorurteil oder von der Mode genarrt, ist die Menge nicht im Stande, das Gute und Solide, sei es ALT oder NEU, von der schwindelhaften oder stümperhaften Mache zu unterscheiden... Wo sehen wir die Ergebnisse der naturwissenschaftlichen Forschung und der hochentwickelten Technik im Alltagsleben völlig wirksam? Jeder Einsichtige gibt ohne weiteres zu, dass das Leben in den Städten durchaus nicht immer die Forderungen der Hygiene erfüllt und dass in sozialer Beziehung viele Erscheinungen sind, die der Ethik schroff widersprechen. [...] Die Hohe Warte, die diese Bewegung aufnimmt und sie in weite Kreise trägt, betont nicht den Gegensatz zwischen modern und unmodern, sondern zwischen GUT UND SCHLECHT ${ }^{21}$.

8 Sa revue réagit aux mutations sociales et technologiques, et l'on perçoit que le progrès, même s'il est validé d'un point de vue scientifique, y est aussi conçu comme un mouvement de dépersonnalisation, comme une perte d'authenticité. Si l'on poursuit le fil implicite de cette réflexion, le mal et le remède viennent d'Angleterre. Lux se destine alors à trouver une réponse aux ravages esthétiques et moraux de la société industrielle de masse, la difficulté essentielle pour chaque nation étant de ne pas perdre son identité au contact des nations voisines. 
9 À quoi ressemble cette revue bimensuelle de 30 pages? Les rubriques se penchent sur tous les aspects de la vie urbaine, de l'organisation de la ville, à la décoration d'intérieur en passant par les arts décoratifs, la politique culturelle et surtout la sauvegarde du patrimoine ${ }^{22}$. Dans chaque numéro un ou plusieurs articles, signés souvent de la main de l'éditeur lui-même, rendent compte des innovations étrangères, des expositions en Allemagne, en Angleterre, en France, au Danemark ou aux EtatsUnis. Une grande partie des contributions est constituée d'extraits d'ouvrages à paraître, comme Das englische Haus de Muthesius, ou les études de Paul SchultzeNaumburg et Adolf Lichtwark. Tous ces reportages illustrés son entrecoupés de citations qui élargissent le cadre de la réflexion, les aphorismes sont signés Morris, Ruskin, Whitman et Wilde. La série la plus caractéristique de la revue se penche sur les mutations urbaines du point de vue de la protection du patrimoine en analysant une région ou une ville de l'Empire des Habsbourg ou du Reich allemand.

10 A cet égard, la revue s'insère parfaitement dans ce mélange de tradition et de modernité que l'on a pu voir comme la caractéristique essentielle de la modernité viennoise $^{23}$. Elle fait le grand écart entre les Arts and Crafts, le fonctionnalisme sécessionniste et la sauvegarde du patrimoine, entre le socialisme progressiste et le particularisme régional ${ }^{24}$. D'ailleurs cette contradiction était déjà le propre de la réflexion de Morris. Anne-Marie Thiesse montre que le mouvement Arts and Crafts naît bien de la critique «de l'asservissement humain par le travail industriel $»^{25}$. En Angleterre, la critique de l'industrialisation se fait sous le signe du retour à la création artisanale, à la tradition nationale. Le progrès esthétique, éthique se pose contre le progrès technique, industriel.

11 Vienne est ainsi replacée dans un cadre plus large, qui mène vers la province et vers l'étranger. La liste des co-éditeurs (plus symbolique que réelle) traduit la complexité des points de vue rassemblés dans la revue. A côté de Muthesius, l'un des grands intermédiaires de l'art anglais en Autriche et en Allemagne, on retrouve Otto Wagner et Josef Hoffmann, les piliers de la Sécession, mais aussi le directeur de la Kunsthalle de Hambourg, Alfred Lichtwark. La Hohe Warte décide de se placer au-delà des écoles, des mouvements esthétiques et nationaux, tout comme le faisait au même moment Deutsche Kunst und Dekoration d'Alexander Koch à Darmstadt. La présence de Lichtwark est révélatrice de cette ouverture, sans pour autant signifier l'arrêt des questionnements identitaires. La comparaison avec le voisin allemand reste l'un des thèmes principaux des articles de Lux et l'on y retrouve les stéréotypes connus qui hantent aussi les écrits de Hofmannsthal ${ }^{26}$.

12 Cette perspective explique aussi la délocalisation de la revue à Leipzig en 1906, lorsque Lux est appelé à participer au projet de la Gartenstadtbewegung ${ }^{27}$. C'est en cela que la revue présente une étude de cas intéressante, où l'on retrouve sous forme condensée la plupart des problèmes auxquels les créateurs viennois ont pu être confrontés au début $\mathrm{du} \mathrm{xx}^{\mathrm{e}}$ siècle. Le pivot de cette problématique se situe dans le rapport à la nation et à l'Etat. Le manque de soutien, de visibilité et l'autocritique permanente d'un Etat considéré comme décadent et coupé de son temps se reflète dans les exils de Bahr ou de Reinhardt. 


\section{Hohe Warte, un pont entre l'Allemagne et l'Autriche?}

Dans les revues d'art et de décoration allemandes, que ce soit la revue conservatrice Kunstwart ou la progressiste Deutsche Kunst und Dekoration d'Alexander Koch, le programme est clairement patriotique et si les revues publient des reportages sur les mouvements anglais, français ou autrichiens, il s'agit pour elles de se défaire d'une influence dite pesante et de stimuler l'esprit de concurrence international, l'impérialisme des arts. Dans le premier numéro de Deutsche Kunst und Dekoration, l'éditeur Alexander $\mathrm{Koch}^{28}$ expose son programme, tout comme Lux quelques années plus $\operatorname{tard}^{29}$, en termes guerriers. Cette dynamique nationale, certes abusive et contreproductive, est bien ce qui semble faire défaut à la couronne autrichienne, si l'on considère tout du moins les articles publiés dans les revues allemandes et viennoises sur la création et son 'émulation' en Autriche-Hongrie. Dans la revue allemande Kunst und Künstler ${ }^{30}$, Franz Servaes regrette en 1903 l'immobilisme politique et social qu'il rend responsable de la crise des arts en Autriche. Si l'esprit créateur existe, il ne trouve pas d'emploi à Vienne et doit se réfugier en Allemagne ${ }^{31}$. Ce constat n'est pas isolé. Pour y remédier, Lux publie d'abord sous forme de feuilleton sa Volkswirtschaft des Talentes en guise de programme de politique culturelle alternative et insiste, dans un article sur les expositions allemandes en 1906, sur le dynamisme germanique. L'article se lit comme un condensé des crises identitaires viennoises et certaines images font directement écho à ce que Hofmannsthal écrira dans ses Lettres du voyageur à son retour. L'Allemagne du modernisme est le révélateur des lacunes autrichiennes :

Jede Deutschlandreise muss für uns Österreicher mit einem Katzenjammer enden. Wir sehen die machtvolle Entfaltung der Kräfte mit Bewunderung und Neid. Das Gefühl unserer Schwäche und Ohnmacht fällt doppelt so schwer auf uns. [...] Sicherlich fehlt ihnen de grazile Anpassung, die das Österreichertum befähigt, manche fremdartige Blüte in den eigenen Topf zu versetzen und damit die Fenster zu schmücken. Im Vergleich zu dieser Beweglichkeit ist deutsche Art allerdings ein schweres Fuhrwerk, aber was das Genialische versagt, hat rastloser Fleiss, unermüdlicher Wille und Mannszucht reichlich ersetzt ${ }^{32}$.

14 L'Autriche y est placée du côté du génie, mais aussi de la médiation : le peuple du milieu, le peuple sans caractère s'adapte, reprend à son compte et transforme les influences étrangères pour leur insuffler une vie nouvelle. Ce stéréotype, que l'on retrouve aussi bien chez Hofmannsthal que dans les articles de Kunst und Kunsthandwerk, fait partie de la 'propagande' habituelle, telle qu'on peut la lire dans l'article de Levetus pour le numéro spécial du Studio en 1906, consacré au renouveau artistique en Autriche ${ }^{33}$. Mais si l'Autrichien se caractérise par sa faculté d'adaptation ${ }^{34}$, son gouvernement ne lui permet pas de développer son talent. Lux continue dans sa comparaison à charge des politiques culturelles allemande et autrichienne :

Der Unterschied wird einem sofort klar, wenn man bedenkt, dass dieser einzige wirkliche Architekt in Österreich nicht einmal in der läppischen KarlsplatzAngelegenheit durchdringen konnte, während in Deutschland Städte und Regierung wetteifern, Jahr für Jahr die Gelder für Monumentalwerke und imposante Kunstausstellungen zusammenzuschiessen, die blutjunge Menschen vor berückende Aufgaben stellen $[. . .]^{35}$.

15 En 1907, Lux finit par tirer les conclusions de son article et délocalise sa revue en Allemagne. Tout d'abord il est appelé par Hermann Muthesius à devenir le 'propagandiste' officiel de la Vereinigte Werkstätte à Munich en 1907, puis un autre membre fondateur du Werkbund, Karl Schmidt, le directeur de la Dresdener Werkstätte für 
Handwerkskunst à Hellerau, l'invite à mettre en place l'école des arts décoratifs ${ }^{36}$. Il se rend à Dresde en 1907 et s'inscrit dans le cadre du mouvement de la Gartenstadt ${ }^{37}$. Lux devient le porte-parole du Werkbund, d'une association de créateurs et d'industriels à Munich qui veut créer en dehors des normes établies : le devenir s'oppose à la norme du système gouvernemental, scolaire. C'est aussi à partir de l'année 1906, où la revue s'ouvre à la Gartenstadtbewegung, que Lux repense l'orientation de sa revue. Il ne s'adresse plus aux masses, mais aux créateurs, aux modernes, aux gens du 'devenir'.

Wir haben für die große Masse kein fertiges Lesefutter zu bieten, und können von vornherein nur mit der Schar von Gleichgesinnten bahnen, mit den Werdenden, die fruchtbare Kräfte entwickeln. Wir maßen uns nicht an Fertiges und Ganzfertiges zu geben. Wir fühlen uns selbst zu sehr im Werden und in der Entwicklung ${ }^{38}$.

Notons que Rudolf Kassner avait développé cette même opposition entre l'être et le devenir dès 1900 dans son dialogue socratique Stil, en l'appliquant à l'identité germanique d'une part et à l'identité anglaise de l'autre ${ }^{39}$ : le génie créatif goethéen s'opposait à la faculté critique anglaise, la pensée au penser. Lux se place ainsi au cœur des débats identitaires propres à la jeune Vienne. Il marque aussi un tournant dans la perception de ce mouvement qui, à partir de 1909, va chercher de nouvelles issues à sa crise.

17 Là encore, Lux est un enfant de son temps. L'expérience allemande au sein du Werkbund se termine dès 1908. Comme le montre Mark Jarzombek, alors que Lux tend de plus en plus vers une sorte de mysticisme catholique, un fossé le sépare dorénavant tant du rationalisme de Muthesius que du patriotisme local des Heimatschutzbewegungen ${ }^{40}$. La revue s'arrête en $1908^{41}$. Ses efforts de rapprochement culturel des deux nations se font encore jour dans une étude au titre révélateur, publiée à Stuttgart en 1915: Der österreichische Bruder. Ein Buch zum Verständnis Österreichs, seiner Menschen, Völker, Schicksale, Städte und Landschaften als Grundlage der geistigen und wirtschaftlichen Annäherung, et, la même année, dans une autre somme programmatique: Deutschland als Welterzieher ${ }^{42}$. Lux, comme cet autre agitateur culturel et auto-propagandiste, Hermann Bahr, rentrera déçu de son exil allemand. Converti au catholicisme en 1925, il se retranche dans un village proche de Salzbourg et nourrit le rêve de transformer cette ville-frontière en un centre culturel dont le rayonnement serait comparable au Weimar de Goethe et Schiller. Ses écrits reflètent cette évolution religieuse et son tournant ultraconservateur ne fait aucun doute. En 1934, il publie DasgoldeneBuchderVaterländischenGeschichte für Volk und Jugend Österreichs. Lapréface est signée d'un certain Kurt Schuschnigg!

\section{NOTES}

1. Carl Schorske, Wien, Geist und Gesellschaft im Fin de siècle, München, Piper, 1982, p. 23-25.

2. Pierre, Bourdieu, Les règles de l'art. Genèse et structure du champ littéraire, Paris, éditions Fayard, 1998.

3. Cf. Yves Chevrefils-Desbiolles, „La dimension internationale des revues d'art", in Des revues d'art en Europe. Ent'revues, Strasbourg, Les Musées de la ville de Strasbourg, 1991, p. 12. 
4. Sylvie Arlaud, „Kunst und Kunsthandwerk: une revue entre modernité viennoise et politique culturelle“, in Revue d'Allemagne et des pays de langue allemande, tome 34, numéro 2, avril-juin 2002, pp. 139-160.

5. Gérard Monnier, L'art et ses institutions en France. De la Révolution à nos jours, Paris, Gallimard, 1995, p. 162-164.

6. Anne-Marie Thiesse, La création des identités nationales. Europe XVIII et XXe siècle, Paris, Éditions du Seuil, 2001, p. 210 : „L'un des principaux enjeux des grandes expositions est le rapport entre art et industrie ou, plus précisément, la conception d'arts appliqués correspondant à l'âge industriel. La valorisation de l'artisanat est en fait indissolublement liée au développement de la production manufacturée. “

7. Ibid., p. 201-204.

8. Wilhelm Bode, Kunst und Kunstgewerbe am Ende des neuzehnten Jahrhunderts, Berlin, Cassirer, 1901, p. 53.

9. Ibid. p. 54: „Wir dürfen uns rühmen, die Engländer in der Zahl der Schulen und Sammlungen für das Kunstgewerbe überflügelt zu haben, während die anderen Nationen gar nicht gegen uns aufkommen können: das wird Stolz bei der Verhandlung jedes Budgets von den Regierungs- und Magistratstischen ausgesprochen und den Landes- und Städtevertretungen dankbar anerkannt." 10. Dominique Poulot, Patrimoine et musées, L'institution de la culture, Paris, Hachette, 2001, p. 77-79.

11. Mark Jarzombek, „Joseph August Lux (1871-1947): Historian of a Lost Modernity“, in Journal of the Society of Architectural Historians 63/2, juin 2004, p. 202-219. Helga Kusolitsch, Hohe Warte, Villenkolonie und Zeitschrift? Die Geschichte einer flüchtigen Begegnung, Wien (Univ. - Diplomarbeit), 1996.

12. Hugo von Hofmannsthal, „Internationale Kunst-Ausstellung 1894“, et „Über moderne englische Malerei“" in Gesammelte Werke in zehn Bänden, Reden und Aufsätze I, Frankfurt/Main, Fischer Taschenbuch Verlag, 1979, p. 534 et p. 546.

13. Karl Panagl, Die britische Kunstzeitschrift The Studio und ihre Bedeutung für die Entwicklung des Jugendstils in Europa, thèse, Vienne, 1972.

14. Jutta Thamer, Zwischen Historismus und Jugendstil. Zur Ausstattung der Zeitschrift Pan (1895-1900), Frankfurt/Main, Peter Lang, 1980.

15. Bernhard Kleinschmidt, Die ,gemeinsame Sendung'. Kunstpublizistik der Wiener Jahrhundertwende, Wien, Paris Peter Lang, 1989, p. 92-93.

16. Professor Josef Hoffmann, „Arbeitsprogramm der Wiener Werkstätte“, in Hohe Warte, tome 1/1904-1905, p. 268.

17. Cité par Helga Kusoltisch, Typoskript : Das Vermächtnis, p. 120 ; Handschriftensammlung ÖNB ser. nov.34.104.

18. Joseph August Lux, „Vorwort des Herausgebers“, in Hohe Warte: tome 1, 1904-1905, p. 1: „Aber trotz einzelner hoher künstlerischer Leistungen, die in so und so vielen Zeitschriften getreulich gebucht werden, ist im Allgemeinen keine Erhöhung des Geschmackes und der künstlerischen Bildung wahrzunehmen. Im Gegenteil.“

19. Mark Jarzombek, op. cit., p. 203: „The figure of Lux thus serves to remind us that the history of modernism was from the start identical to the history of the response to modernism."

20. Le tirage reste cependant limité, 3000 exemplaires en 1906 contre 22000 pour la revue d'art germanophone la plus lue en 1908.

21. Joseph August Lux, „Vorwort des Herausgebers“, in Hohe Warte: année 1, 1904-1905, p. 1.

22. Ibid.

23. Jacques Le Rider, Modernité viennoise et crises de l'identité, Paris, PUF, 1990.

24. Joseph August Lux, „Nationale Politik“, in Hohe Warte, année 3, 1906-1907, p. 57: „Was es immer für Anstrengung kostet, es wird dahin kommen müssen, dass die Arbeit wieder eine 
Entfaltung edler Menschlichkeit darstellt und das Land und das Leben des Volkes mit den Zeichen des Glücks und der nationalen Gesundheit verschönert.“

25. Anne-Marie Thiesse, op. cit., p. 213.

26. Adolf Lichtwark, „Kunstpolitik“, in Hohe Warte, année 1, 1904-1905, p. 88.

27. Anne-Marie Thiesse, op. cit., p. 252.

28. Cité par Maria Rennhofer, Kunstzeitschriften der Jahrhundertwende in Deutschland und Österreich, 1895-1914, München, Wien, Brandstaetter, 1997, p. 91.

29. Joseph August Lux, „Das Bundesziel“, in Hohe Warte année 3, 1906-1907, p. 297: „Der Wettstreit der Völker entwickelt sich aus dem der physischen Kräfte, wie er sich in den früheren Kriegen äußerte, immer mehr $\mathrm{zu}$ einem Wettstreite der Intelligenz und der Charaktereigenschaften. In der Industrie wird jenem Volke die Zukunft gehören, das die best erzogenen Augen und die edelste Auffassung der Arbeit besitzt. Gerade Deustchland, das politisch eingeengt und in seiner Landerweiterung gehemmt ist, bedarf der Produktionsgebiete, die im Adel der Arbeit ihresgleichen suchen."

30. La revue paraît entre 1902-1933 aux éditions Cassirer, les rédacteurs en chef de la première année sont Emil Heilbut et Cäsar Flaischlen.

31. Franz Servaes, „Die Wiener Kunstgewerbeschule“ in Kunst und Künstler, Berlin, 1903, p. 431-440: „Man hat in Deutschland kaum eine Vorstellung davon, welch eine Fülle von Talent sich aus dieser Schule zusammendrängt, wie bei den Lehrern so auch unter den Lernenden. Österreichs Menschenreichtum auf diesem Gebiet ist einfach Staunen erregend. Also wohin damit [den Ausgebildeten]? Doch die Welt ist groß, und vermutlich wird wohl zuerst Deutschland den guten Magen haben, von der edlen Zufuhr, die das Schwesterland bereit hält, zu verdauen. Deutschland gibt ja so viele und tüchtige Kulturelemente an Österreich ab. Hier ist ein Punkt, wo es von ihm zurückempfangen darf."

32. Joseph August Lux, „Von deutschen Kunstausstellungen 1906“ in Hohe Warte, année 2, 1905-1906, p. 303-306.

33. A.S. Levetus, "Modern Decorative Art in Austria", The Studio, The Art-Revival in Austria, Special Number, Summer 1906, London, Paris, New York, édité par Charles Holme, pp. XI-XII: „A true feeling for art and decoration is inborn in the Austrians. Their national art is sufficient proof of this. But the modern movement in decorative art owes its inception to outside influence - to England ; its development however, comes from the Austrians themselves."

34. Mark Jarzombek, op. cit., p. 207.

35. Joseph August Lux, „Von deutschen Kunstausstellungen 1906“ in Hohe Warte, année 2, 1905-1906, p. 303.

36. Mark Jarzombek, op. cit., p. 215.

37. Anne-Marie Thiesse, op. cit., p. 254.

38. Joseph August Lux, „Vorwort zum dritten Jahrgang“, in Hohe Warte, année 3, 1906-1907, p. 1. 39. Rudolf Kassner, „Stil, ein Dialog“, in Sämtliche Werke Band I, Pfullingen, Neske, 1969, p. 303: „Die englischen Dichter sind die groben Verherrlicher des Seins, und die Deutschen haben in ihren wahrsten Gedichten das Werden gefeiert. Das Sein nun besitzt die Musik in sich. Die Musik steht außerhalb der Unruhe des Werdens.“

40. Mark Jarzombek, op. cit., p. 216.

41. Ibid.

42. Les deux ouvrages sont publiés à Stuttgart, aux éditions: Union deutsche Verlagsgesellschaft en 1915. 


\section{RÉSUMÉS}

Les revues d'art et de décoration sont autant les produits que les révélateurs des mutations profondes qui vont traverser les arts et les modes de vie européens au tournant $\mathrm{du} \mathrm{xx}^{\mathrm{e}}$ siècle. Tout comme dans les autres capitales européennes, à Vienne, les journaux et les revues spécialisées dans les arts et la littérature, les musées et les théâtres, connaissent un essor fulgurant sous l'influence libérale dès les années 1860. Ils se développent comme des centres d'opinion en rivalité ou en collaboration avec le pouvoir dynastique. Dans le paysage déjà très saturé des publications d'art apparaît en 1904 une revue symptomatique des contraintes imposées aux artistes et aux critiques en Autriche: la Hohe Warte. L'histoire de cette revue, établie comme un pont étrange entre l'Allemagne et l'Autriche, entre l'art et l'industrie, la ville et la province, ainsi que la biographie originale de son éditeur et principal contributeur, Joseph August Lux, permettent de retracer, au croisement entre art autonome et hétéronome, les principales contradictions de cette modernité viennoise dans son rapport aux autres capitales européennes. Lux et sa revue se lisent comme le condensé de tous les combats et de toutes les illusions perdues d'une génération et d'une «nation » en crise. Derrière Lux, c'est le modèle de Hofmannsthal ou de Bahr qui se profile.

Die Kunstzeitschriften gewähren einen Einblick in die gewaltigen Umbrüche, die das Geistesleben und die Künste der europäischen Kulturstädte im 20. Jahrhundert erschüttern und erneuern werden. Ebenso wie in vielen anderen europäischen Hauptstädten, entwickeln sich in Wien unter dem Einfluss der liberalen Ära Zeitungen, Kunstzeitschriften, Museen und Theater in den Jahren um 1860. Sie werden zu Meinungszentren, die mit der Krone entweder rivalisieren oder zusammenarbeiten. In der ohnehin schon überfüllten Zeitschriftenlandschaft erscheint im Jahre 1904 eine seltsame kleine Zeitschrift, deren Geschichte für die Situation des Verlagswesens im untergehenden Habsburger Reich beispielhaft ist: die Hohe Warte, die eine Gratwanderung zwischen Österreich und Deutschland, zwischen Kunst und Industrie, zwischen Stadtleben und Provinzleben zu vollziehen versucht. In ihr und in der Biographie ihres Herausgebers, Joseph August Lux, finden sich die interessantesten Widersprüche der Wiener Moderne wieder. Sie liest sich wie der Schnappschuss einer Zeit und einer ,schwierigen' Nation. Lux' Werdegang liest sich nicht zufällig wie eine Reminiszenz an Hofmannsthals oder Bahrs eigene Entwicklungen nach der Jahrhundertwende.

\section{INDEX}

Mots-clés : revues d'art

oeuvrecitee Die Hohe Warte

\section{AUTEURS}

SYLVIE ARLAUD

Université Lyon II 